This document is the accepted manuscript version of the following article:

Mishra, S., Beyer, D., Berger, R., Liu, J., Gröning, 0., Urge1, J. I., ... Fase1, R. (2020). Topological defect-induced magnetism in a nanographene. Journal of the American Chemical

Society. https://doi.org/10.1021/jacs.9b09212

\title{
Topological defect-induced magnetism in a nanographene
}

\author{
Shantanu Mishra ${ }^{\dagger}$, Doreen Beyer ${ }^{\S}$, Reinhard Berger ${ }^{\S}$, Junzhi Liu ${ }^{\S}$, Oliver Gröning ${ }^{\dagger}$, José I. Urgel ${ }^{\dagger}$, Klaus \\ Müllen", Pascal Ruffieux ${ }^{\dagger}$, Xinliang Feng ${ }^{\S, *}$ and Roman Fasel ${ }^{\dagger, \ddagger}, *$ \\ †nanotech@surfaces Laboratory, Empa - Swiss Federal Laboratories for Materials Science and Technology, Dübendorf, \\ Switzerland \\ ${ }^{\S}$ Faculty of Chemistry and Food Chemistry, and Center for Advancing Electronics Dresden, Technical University of Dres- \\ den, Dresden, Germany \\ IDepartment of Synthetic Chemistry, Max Planck Institute for Polymer Research, Mainz, Germany \\ *Department of Chemistry and Biochemistry, University of Bern, Bern, Switzerland
}

\begin{abstract}
The on-surface reactions of 10-bromo-10'-(2,6dimethylphenyl)-9,9'-bianthracene on $\mathrm{Au}(111)$ have been investigated by scanning tunneling microscopy and spectroscopy, complemented by theoretical calculations. The reactions afford synthesis of two open-shell nanographenes (1a and 1b) exhibiting different scenarios of all-carbon magnetism. 1a, an all-benzenoid nanographene with triangulene-like termini, contains a high proportion of zigzag edges which endows it with a low frontier gap and edge-localized states. The dominant reaction product $(\mathbf{1 b})$ is a non-benzenoid nanographene consisting of a single pentagonal ring in a benzenoid framework. The presence of this nonbenzenoid topological defect, which alters the bond connectivity in the hexagonal lattice, results in a non-Kekule nanographene with an unpaired spin, which is detected as a Kondo resonance.
\end{abstract}

Inducing magnetism in carbon nanomaterials has been a longstanding challenge encompassing organic chemistry and condensed matter physics. ${ }^{1}$ Owing to lower spin-orbit and hyperfine couplings, ${ }^{1,2}$ and larger spin correlation lengths, ${ }^{3}$ carbon nanomaterials are postulated to exhibit improved performance over inorganic materials in spin-based information processing. ${ }^{1,4,5}$ The field of carbon magnetism has received a revived interest since developments in the bottom-up synthetic chemistry of polycyclic conjugated hydrocarbons, or nanographenes (NGs). ${ }^{6}$ The electronic structure of NGs exhibits sensitive dependence on the topology of the $\pi$-electron network, which provides a platform to engineer a plethora of properties, including magnetism. The high reactivity of magnetic (open-shell) NGs makes a conventional solutionbased synthesis challenging, and in this regard, on-surface synthesis ${ }^{7}$ under ultrahigh vacuum has emerged as a powerful alternative synthetic toolbox. Current progress in the synthesis of open-shell NGs is largely limited to low-spin NGs with extended zigzag edges $^{8-21}$ or high-spin non-Kekulé NGs. ${ }^{22-27}$ However, much less attention has been paid on the potential of topological defects, that is, non-benzenoid rings, in inducing magnetism in NGs. Nonbenzenoid rings, routinely observed as defects in graphene, ${ }^{28}$ may significantly influence the chemical ${ }^{29}$ and electronic properties $^{16,30-32}$ as a result of local changes in strain and conjugation. Here, we report a combined in-solution and on-surface synthesis of two open-shell NGs resulting from on-surface reactions of a single molecular precursor 10-bromo-10'-(2,6-dimethylphenyl)9,9'-bianthracene (2, Scheme 1) on $\mathrm{Au}(111)$, and their characterization using scanning tunneling microscopy (STM) and spectroscopy (STS), supported by tight-binding (TB) and mean-field Hubbard (MFH) calculations. Surface-catalyzed Ullmann coupling and subsequent cyclodehydrogenation and oxidative cyclization reactions of $\mathbf{2}$ leads to the formation of two distinct NGs. 1a, an all-benzenoid NG formed in minority, results from a defect-free on-surface reaction of $\mathbf{2}$. It consists of triangulene-like termini which present extended zigzag edges hosting localized states with an antiferromagnetic coupling across the NG, and an exceedingly low frontier gap of $110 \mathrm{meV}$. Interestingly, a majority of Ullmann-coupled dimers of $\mathbf{2}$ lose a single methyl group, leading to the formation of a pentagonal ring in an all-benzenoid framework (1b). The presence of this topological defect renders 1b a non-Kekulé NG with an unpaired electron, which manifests as a Kondo resonance. ${ }^{33}$

The solution synthesis of 2 (Scheme 1) commenced with the formation of 9,9'-bianthracene (4), derived from commercially available 9,10-anthraquinone (3). The synthesis was carried out according to literature protocol, ${ }^{34}$ and $\mathbf{4}$ was isolated as a yellow solid in moderate yield. Bromination of $\mathbf{4}$ at low temperature afforded 10,10'-dibromo-9,9'-bianthracene (5). 5 further reacted with (2,6-dimethylphenyl)boronic acid under Suzuki cross coupling conditions and provided 10-(2,6-dimethylphenyl)-9,9'bianthracene (6) in a mixture of three byproducts, namely 9,9 'bianthracene (6-1), 10-bromo-9,9'-bianthracene (6-2) and 10,10'bis(2,6-dimethylphenyl)-9,9'-bianthracene (6-3) (see Supporting Information). Owing to similar polarities of 6 and 6-1 to 6-3, isolation of 6 by silica gel chromatography was hampered. Thus, the roughly purified compound mixture $\mathbf{6}$ was directly brominated at $0{ }^{\circ} \mathrm{C}$, and $\mathbf{2}$ was obtained as a light-sensitive yellow solid with an overall yield of $5 \%$.

Subsequently, a submonolayer coverage of $\mathbf{2}$ was deposited on a $\mathrm{Au}(111)$ surface held at room temperature, and annealed to 300 ${ }^{\circ} \mathrm{C}$ to promote on-surface reactions. Figure 1a presents an overview STM image of the surface, where individual molecules and covalently-bonded oligomers (Figure S1) are found. Highresolution (HR) STM imaging at low tunneling bias reveals that $6 \%$ of the molecules (1a) exhibit a two-lobed local density of states (LDOS) distribution at the termini (Figure 1b). We performed ultrahigh-resolution (UHR) STM imaging to gain access 
In solution:<smiles>O=C1c2ccccc2C(=O)c2ccccc21</smiles>

3<smiles>c1ccc2c(-c3c4ccccc4cc4ccccc34)c3ccccc3cc2c1</smiles>

4
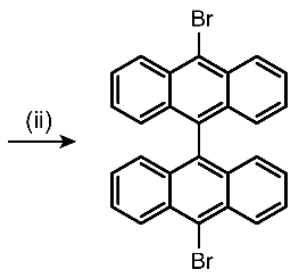

5<smiles>Cc1cccc(C)c1-c1c2ccccc2c(-c2c3ccccc3cc3ccccc23)c2ccccc12</smiles>

$\stackrel{\text { (iv) }}{\longrightarrow}$<smiles>Cc1cccc(C)c1-c1c2ccccc2c(-c2c3ccccc3c(Br)c3ccccc23)c2ccccc12</smiles>

2
On surface:<smiles>Cc1cccc(C)c1-c1c2ccccc2c(-c2c3ccccc3c(Br)c3ccccc23)c2ccccc12</smiles>

2
Closed-shell Kekulé structure, 6 Clar sextets

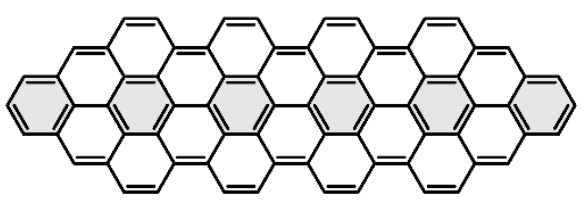

1a, all-benzenoid low-gap nanographene

\section{$\mathrm{Au}(111), 300^{\circ} \mathrm{C}$}

1. Ullmann coupling 2. Cyclodehydrogenation and oxidative cyclization

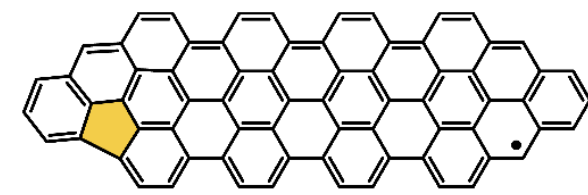

1b, non-benzenoid non-Kekulé nanographene
Open-shell non-Kekulé structure, 9 Clar sextets

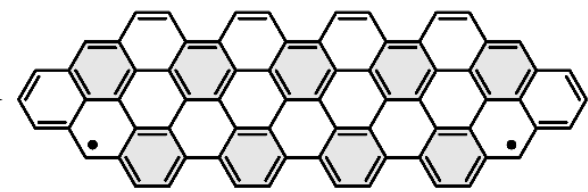
. 


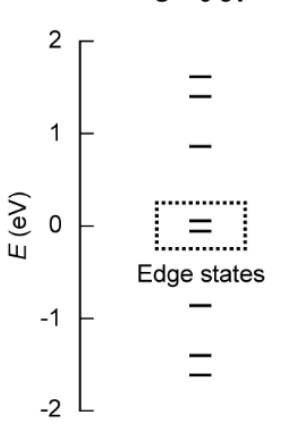

g
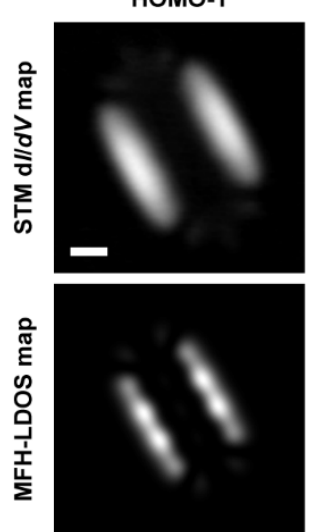

b

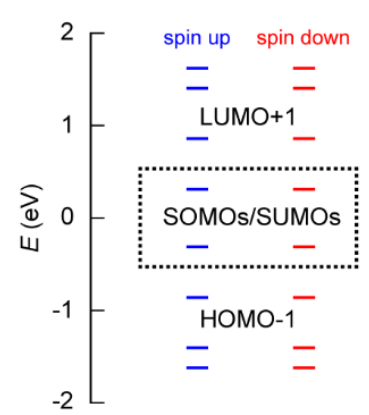

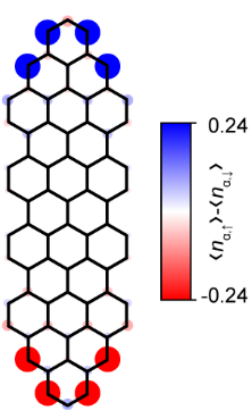

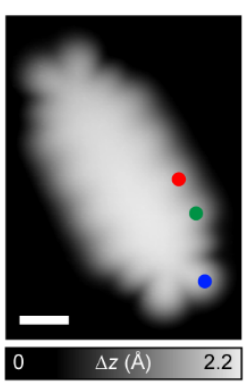

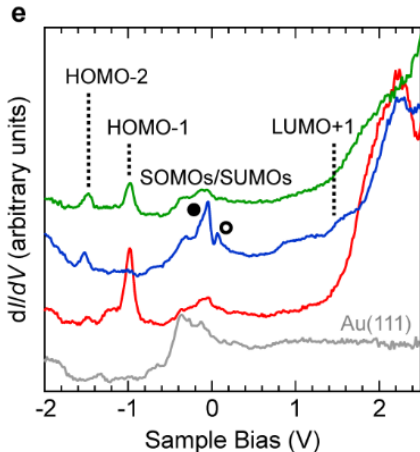

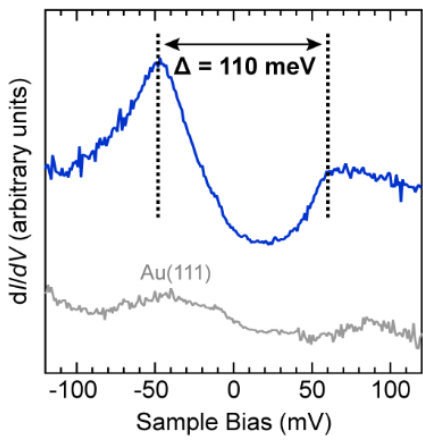

Figure 2. Electronic characterization of 1a. $(a, b)$ Nearest-neighbor TB (a) and MFH (b) energy spectrum of 1a. (c) MFH spin density distribution of 1a. Blue/red isosurfaces denote spin up/spin down density. (d) HR-STM image of 1a. (e, f) dI/dV spectroscopy on 1a. Acquisition positions are marked with corresponding filled circles in (d). The spectra in all panels are vertically offset for visual clarity. (g) Constant-current $\mathrm{d} I / d V$ maps (top panels) and simulated MFH-LDOS maps (bottom panels) of the HOMO-1, SOMOs, SUMOs and LUMO +1 of 1a. Scanning parameters: $V=-30 \mathrm{mV}, I=350 \mathrm{pA}(\mathrm{d}) ; V=-980 \mathrm{mV}, I=400 \mathrm{pA}(\mathrm{g}, \mathrm{HOMO}-1) ; V=-30 \mathrm{mV}, I=350 \mathrm{pA}(\mathrm{g}$, SOMOs); $V=+50 \mathrm{mV}, I=400 \mathrm{pA}\left(\mathrm{g}\right.$, SUMOs) and $V=+1.45 \mathrm{~V}, I=400 \mathrm{pA}(\mathrm{g}, \mathrm{LUMO}+1) ; V_{\mathrm{rms}}=10 \mathrm{mV}$ (g, SOMOs and SUMOs) and $22 \mathrm{mV}$ (g, HOMO-1 and LUMO+1). Open feedback parameters for $\mathrm{d} I / d V$ spectra: $V=-2.00 \mathrm{~V}, I=370 \mathrm{pA}(\mathrm{e})$ and $V=-50 \mathrm{mV}, I=500 \mathrm{pA}$ (f); $V_{\mathrm{rms}}=16 \mathrm{mV}$ (e) and $2 \mathrm{mV}$ (f). Scale bars: $0.5 \mathrm{~nm}$.

ure 1f). Figure $1 \mathrm{~g}$ presents the UHR-STM image of $\mathbf{1 b}$, revealing strong convolution of chemical structure with LDOS near zero bias at the lower terminus, in contrast to the upper terminus which does not exhibit any zero-bias LDOS. UHR-STM imaging of the termini shows the formation of a pentagonal ring at one of the termini (Figure 1h), with the other terminus retaining the triangulene-like structure (Figure 1i). Notably, the zero-bias LDOS accumulates almost exclusively on the triangulene-like terminus. Evidently, formation of a pentagonal ring implies the loss of a single methyl group from an Ullmann-coupled dimer of 2 (we rarely detect molecules with the loss of two methyl groups, see Figure S2)

To elucidate the electronic structures of the molecules, we employ the nearest-neighbor TB model. Figure 2a and Figure S3 present the TB energy spectrum of 1a, which shows a pair of states located close to zero energy. These states are localized at the termini with a fast decay of wave function amplitude toward the center of the molecule, characteristic of edge states $^{37}$ (Figure S4). To account for electron-electron interactions, we employ the MFH model, which leads to spin polarization in the system. The frontier electronic structure is characterized by a pair of singly occupied molecular orbitals (SOMOs, Figure 2b), with the populating spins aligned antiferromagnetically (Figure S5), in agreement with Ovchinnikov's rule ${ }^{38}$ and previous calculations. ${ }^{39}$ The computed spin density distribution of $\mathbf{1 a}$ (Figure 2c) reveals dominant spin localization at the zigzag carbon atoms at the termini, with spin up and spin down populations spatially separated. The experimental electronic structure of $\mathbf{1 a}$ is characterized via STS, which reproduces the salient features of the TB/MFH calculations. As shown in Figure 2d,e, d $I / d V$ spectroscopy on 1a reveals a series of electronic resonances labeled HOMO-2 (-1.50 V; HOMO is highest occupied molecular orbital), HOMO-1 (-980 mV) and LUMO $+1(+1.45 \mathrm{~V}$; LUMO is lowest unoccupied molecular orbital), along with a broad peak at $+2.23 \mathrm{~V}$. Additionally, a pair of electronic resonances near zero bias are detected (Figure 2e, marked with filled and empty circles and labeled SOMOs/SUMOs; SUMO is singly unoccupied molecular orbital). $\mathrm{d} I / d V$ spectroscopy in the vicinity of the Fermi level reveals two peaks at $-50 \mathrm{mV}$ and $+60 \mathrm{mV}$ (Figure $2 \mathrm{f}$ ). Spatial mapping of the $\mathrm{d} I / d V$ signal $(\mathrm{d} I / d V$ maps) around $-980 \mathrm{mV},-50 \mathrm{mV}$ and $+60 \mathrm{mV}$ (Figure 2g) exhibits excellent agreement with the mean-field Hubbard local density of states (MFH-LDOS) maps of the HOMO-1, SOMOs and SUMOs of 1a, respectively. This confirms the spectroscopic features labeled in Figure 2e as molecular orbital resonances (see Figures S6 and S7 for additional STS data on 1a). Based on the energies of the SOMOs and SUMOs, the frontier gap of 1a is inferred to be $110 \mathrm{meV}$. We note that the $\mathrm{d} I / d V$ map acquired at $+1.45 \mathrm{~V}$, while exhibiting partial agreement with the LUMO+1 MFH-LDOS map, presents prominent maxima at the center of the armchair edges - in contrast to the weak LDOS expected for LUMO+1 at this location. This observation may be explained by a weak intermixing of $\mathrm{LUMO}+1$ and $\mathrm{LUMO}+2$ states (Figure S8). Finally, an open question pertaining to the experimental electronic structure of $\mathbf{1 a}$ is the absence of inelastic spin excitation signal ${ }^{40}$ in $\mathrm{d} I / d V$ spectroscopy. Given the $S=0$ ground state of $\mathbf{1 a}$ (where $S$ is the total spin quantum number), an 

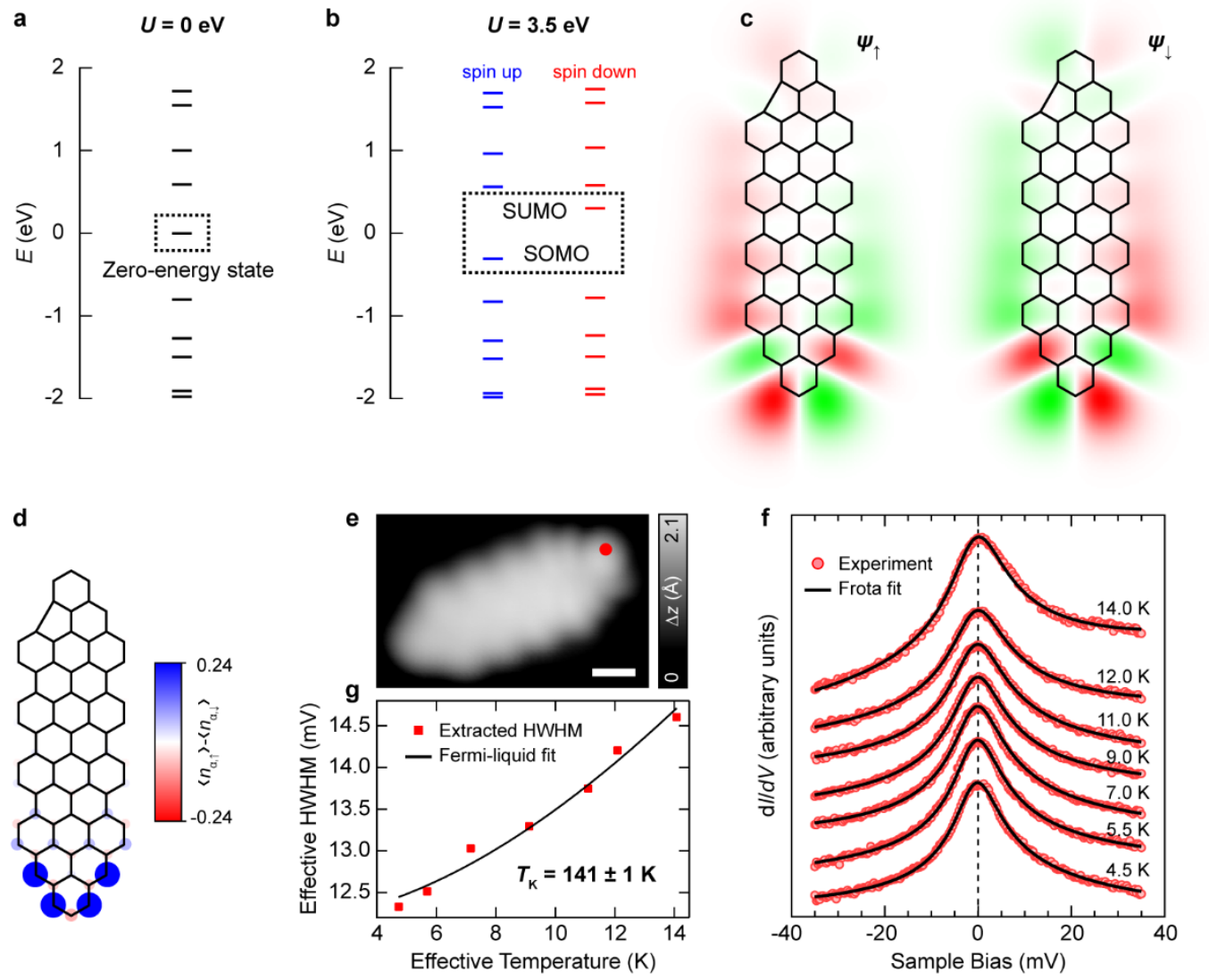

Figure 3. Electronic characterization of $\mathbf{1 b}$. (a, b) Nearest-neighbor TB (a) and MFH (b) energy spectrum of 1b. (c) Computed wave functions of the SOMO $(\psi \uparrow)$ and SUMO $\left(\psi_{\downarrow}\right)$ of $\mathbf{1 b}$. Green/red isosurfaces denote opposite phase of the wave function. (d) MFH spin density distribution of $\mathbf{1 b}$. (e) HR-STM image of $\mathbf{1 b}$ (repeated from Figure 1f). (f) Temperature evolution of the Kondo resonance detected in 1b, with corresponding fit using the Frota function. ${ }^{41}$ Acquisition position is marked with a filled circle in (e). (g) Temperature evolution of the extracted half width at half maximum (HWHM) of the Kondo resonance, with fit using the Fermi-liquid model. ${ }^{33}$ Open feedback parameters for $\mathrm{d} I / d V$ spectra: $V=-35 \mathrm{mV}, I=300 \mathrm{pA}, V_{\mathrm{rms}}=400 \mu \mathrm{V}$. Scale bars: $0.5 \mathrm{~nm}$.

$S=0$ to $S=1$ (that is, singlet-triplet) spin excitation is to be expected $^{20,21}$. We speculate that since tails of the broad SOMOs and SUMOs resonances persist up to the Fermi energy (Figure 2f), this large contribution from an elastic tunneling channel may obscure the weaker inelastic features.

In contrast to the all-benzenoid topology of $1 \mathbf{a}$, the presence of a pentagonal ring in $\mathbf{1 b}$ disrupts the bipartite symmetry of the underlying honeycomb lattice, and Ovchinnikov's rule cannot be applied to determine the ground state spin multiplicity. Importantly, presence of the pentagonal ring renders $\mathbf{1 b}$ a non-Kekulé system with an unpaired electron, that is, $S=1 / 2$. Accordingly, the TB energy spectrum of $\mathbf{1 b}$ (Figure $3 \mathrm{a}$ ) reveals a zero-energy state occupied by one electron. Inclusion of on-site Coulomb repulsion triggers spin polarization, along with the opening of a Coulomb gap and a net spin imbalance of one in the system (Figure 3b). The computed spin-polarized wave functions of the SOMO and SUMO of $\mathbf{1 b}$ is displayed in Figure 3c, which exhibit dominant localization at the triangulene-like terminus, with negligible amplitude at the pentagonal ring-containing terminus. The computed spin density distribution of $\mathbf{1 b}$ (Figure $3 \mathrm{~d}$ ) reveals spin localization only at the triangulene-like terminus, in agreement with the spatial extent of the zero-bias LDOS (Figure $1 \mathrm{~g}$ ). The hallmark of a spin- $1 / 2$ system on a metal surface is screening of the individual spin by itinerant conduction electrons of the surface, leading to a many-body Kondo ground state. $\mathrm{d} I / d V$ spectroscopy on $\mathbf{1 b}$ reveals a pronounced zero-bias peak which exhibits an anomalous resonance linewidth broadening with increasing temperature, following the characteristic trend of a Kondo resonance with a Kondo temperature $T_{\mathrm{K}}=141 \mathrm{~K}$ (Figure 3e-g; also see Figures $\mathrm{S} 9$ and $\mathrm{S} 10$ ), unambiguously establishing spin- $1 / 2$ paramagnetism.

In summary, we have shown synthesis of two open-shell NGs, $\mathbf{1 a}$ and $\mathbf{1 b}$, resulting from the on-surface reactions of a single precursor $\mathbf{2}$ on $\mathrm{Au}(111)$. 1a is an all-benzenoid NG containing zigzag edge-rich termini hosting low-energy edge states, with a frontier electronic gap of $110 \mathrm{meV}$. 1b is a non-benzenoid NG with a single pentagonal ring in a benzenoid framework. Presence of this topological defect renders $\mathbf{1 b}$ a non-Kekulé system with $S$ $=1 / 2$, which is detected as a Kondo resonance. Our work demonstrates unambiguous evidence of all-carbon magnetism, and provides rich opportunities to induce and tune magnetism in NGs through topological defects.

\section{ASSOCIATED CONTENT}

\section{Supporting Information}

The Supporting Information is available free of charge on the ACS Publications website.

Additional TB and MFH calculations, and STM/STS data (Figures S1-S10), detailed synthetic procedures of chemical compounds reported in this study (Schemes S1-S4), associated solution characterization data (Figures S11-S17), mathematical fit procedure for Kondo resonance, and experimental and calculation methods (PDF)

\section{AUTHOR INFORMATION}




\section{Corresponding Author}

*xinliang.feng@tu-dresden.de

*roman.fasel@empa.ch

\section{Notes}

The authors declare no competing financial interests.

\section{ACKNOWLEDGMENT}

This work was supported by the Swiss National Science Foundation (grant numbers 200020-182015 and IZLCZ2-170184), the European Union's Horizon 2020 research and innovation program under grant agreement number 785219 (Graphene Flagship Core 2), the Office of Naval Research (grant number N00014-18-12708), ERC Consolidator grant (T2DCP, number 819698), the German Research Foundation (DFG) within the Cluster of Excellence - Center for Advancing Electronics Dresden (cfaed) and EnhanceNano (number 391979941), and the European Social Fund and the Federal State of Saxony (ESF-Project GRAPHD, TU Dresden).

\section{REFERENCES}

(1) Yazyev, O. V. Emergence of Magnetism in Graphene Materials and Nanostructures. Rep. Prog. Phys. 2010, 73 (5), 056501.

(2) Trauzettel, B.; Bulaev, D. V.; Loss, D.; Burkard, G. Spin Qubits in Graphene Quantum Dots. Nat. Phys. 2007, 3 (3), 192-196.

(3) Yazyev, O. V.; Katsnelson, M. I. Magnetic Correlations at Graphene Edges: Basis for Novel Spintronics Devices. Phys. Rev. Lett. 2008, 100 (4), 047209

(4) Wang, W. L.; Yazyev, O. V.; Meng, S.; Kaxiras, E. Topological Frustration in Graphene Nanoflakes: Magnetic Order and Spin Logic Devices. Phys. Rev. Lett. 2009, 102 (15), 157201.

(5) Bullard, Z.; Girão, E. C.; Owens, J. R.; Shelton, W. A.; Meunier, V. Improved All-Carbon Spintronic Device Design. Sci. Rep. 2015, 5, 7634.

(6) Narita, A.; Wang, X.-Y.; Feng, X.; Müllen, K. New Advances in Nanographene Chemistry. Chem. Soc. Rev. 2015, 44 (18), 6616-6643.

(7) On-Surface Synthesis; Gourdon, A., Ed.; Springer International Publishing: Cham, 2016.

(8) Konishi, A.; Hirao, Y.; Nakano, M.; Shimizu, A.; Botek, E.; Champagne, B.; Shiomi, D.; Sato, K.; Takui, T.; Matsumoto, K.; Kurata, H.; Kubo, T. Synthesis and Characterization of Teranthene: A Singlet Biradical Polycyclic Aromatic Hydrocarbon Having Kekulé Structures. J. Am. Chem. Soc. 2010, 132 (32), 11021-11023.

(9) Konishi, A.; Hirao, Y.; Matsumoto, K.; Kurata, H.; Kishi, R.; Shigeta, Y.; Nakano, M.; Tokunaga, K.; Kamada, K.; Kubo, T. Synthesis and Characterization of Quarteranthene: Elucidating the Characteristics of the Edge State of Graphene Nanoribbons at the Molecular Level. J. Am. Chem. Soc. 2013, 135 (4), 1430-1437.

(10) Tönshoff, C.; Bettinger, H. F. Photogeneration of Octacene and Nonacene. Angew. Chem. Int. Ed. 2010, 49 (24), 4125-4128.

(11) Krüger, J.; García, F.; Eisenhut, F.; Skidin, D.; Alonso, J. M.; Guitián, E.; Pérez, D.; Cuniberti, G.; Moresco, F.; Peña, D. Decacene: OnSurface Generation. Angew. Chem. Int. Ed. 2017, 56 (39), 11945-11948.

(12) Zuzak, R.; Dorel, R.; Kolmer, M.; Szymonski, M.; Godlewski, S.; Echavarren, A. M. Higher Acenes by On-Surface Dehydrogenation: From Heptacene to Undecacene. Angew. Chem. Int. Ed. 2018, 57 (33), 10500 10505 .
(13) Eisenhut, F.; Kühne, T.; García, F.; Fernández, S.; Guitián, E.; Pérez, D.; Trinquier, G.; Cuniberti, G.; Joachim, C.; Peña, D.; Moresco, F. Dodecacene Generated on Surface: Reopening of the Energy Gap. ACS Nano 2019. https://doi.org/10.1021/acsnano.9b08456.

(14) Urgel, J. I.; Mishra, S.; Hayashi, H.; Wilhelm, J.; Pignedoli, C. A.; Giovannantonio, M. D.; Widmer, R.; Yamashita, M.; Hieda, N.; Ruffieux, P.; Yamada, H.; Fasel, R. On-Surface Light-Induced Generation of Higher Acenes and Elucidation of Their Open-Shell Character. Nat. Commun. 2019, 10 (1), 861

(15) Ajayakumar, M. R.; Fu, Y.; Ma, J.; Hennersdorf, F.; Komber, H.; Weigand, J. J.; Alfonsov, A.; Popov, A. A.; Berger, R.; Liu, J.; Müllen, K.; Feng, X. Toward Full Zigzag-Edged Nanographenes: Peri-Tetracene and Its Corresponding Circumanthracene. J. Am. Chem. Soc. 2018, 140 (20), 6240-6244.

(16) Mishra, S.; Lohr, T. G.; Pignedoli, C. A.; Liu, J.; Berger, R.; Urgel, J. I.; Müllen, K.; Feng, X.; Ruffieux, P.; Fasel, R. Tailoring Bond Topologies in Open-Shell Graphene Nanostructures. ACS Nano 2018, 12 (12), 11917-11927.

(17) Rogers, C.; Chen, C.; Pedramrazi, Z.; Omrani, A. A.; Tsai, H.-Z.; Jung, H. S.; Lin, S.; Crommie, M. F.; Fischer, F. R. Closing the Nanographene Gap: Surface-Assisted Synthesis of Peripentacene from 6,6'Bipentacene Precursors. Angew. Chem. Int. Ed. 2015, 54 (50), 1514315146.

(18) Zeng, W.; Gopalakrishna, T. Y.; Phan, H.; Tanaka, T.; Herng, T. S.; Ding, J.; Osuka, A.; Wu, J. Superoctazethrene: An Open-Shell Graphenelike Molecule Possessing Large Diradical Character but Still with Reasonable Stability. J. Am. Chem. Soc. 2018, 140 (43), 14054-14058.

(19) Ruffieux, P.; Wang, S.; Yang, B.; Sánchez-Sánchez, C.; Liu, J.; Dienel, T.; Talirz, L.; Shinde, P.; Pignedoli, C. A.; Passerone, D.; Dumslaff, T.; Feng, X.; Müllen, K.; Fasel, R. On-Surface Synthesis of Graphene Nanoribbons with Zigzag Edge Topology. Nature 2016, 531 (7595), 489-492.

(20) Li, J.; Sanz, S.; Corso, M.; Choi, D. J.; Peña, D.; Frederiksen, T.; Pascual, J. I. Single Spin Localization and Manipulation in Graphene Open-Shell Nanostructures. Nat. Commun. 2019, 10 (1), 200.

(21) Mishra, S.; Beyer, D.; Eimre, K.; Kezilebieke, S.; Berger, R.; Gröning, O.; Pignedoli, C. A.; Müllen, K.; Liljeroth, P.; Ruffieux, P.; Feng, X.; Fasel, R. Topological Frustration Induces Unconventional Magnetism in a Nanographene. Nat. Nanotechnol. 2019 https://doi.org/10.1038/s41565-019-0577-9.

(22) Morita, Y.; Suzuki, S.; Sato, K.; Takui, T. Synthetic Organic Spin Chemistry for Structurally Well-Defined Open-Shell Graphene Fragments. Nat. Chem. 2011, 3 (3), 197-204.

(23) Goto, K.; Kubo, T.; Yamamoto, K.; Nakasuji, K.; Sato, K.; Shiomi, D.; Takui, T.; Kubota, M.; Kobayashi, T.; Yakusi, K.; Ouyang, J. A. A Stable Neutral Hydrocarbon Radical: Synthesis, Crystal Structure, and Physical Properties of 2,5,8-Tri-tert-butyl-phenalenyl. J. Am. Chem. Soc. 1999, 121 (7), 1619-1620.

(24) Inoue, J.; Fukui, K.; Kubo, T.; Nakazawa, S.; Sato, K.; Shiomi, D.; Morita, Y.; Yamamoto, K.; Takui, T.; Nakasuji, K. The First Detection of a Clar's Hydrocarbon, 2,6,10-Tri-tert-Butyltriangulene: A Ground-State Triplet of Non-Kekulé Polynuclear Benzenoid Hydrocarbon. J. Am. Chem. Soc. 2001, 123 (50), 12702-12703.

(25) Pavliček, N.; Mistry, A.; Majzik, Z.; Moll, N.; Meyer, G.; Fox, D. J.; Gross, L. Synthesis and Characterization of Triangulene. Nat. Nanotechnol. 2017, 12 (4), 308-311.

(26) Mishra, S.; Beyer, D.; Eimre, K.; Liu, J.; Berger, R.; Gröning, O.; Pignedoli, C. A.; Müllen, K.; Fasel, R.; Feng, X.; Ruffieux, P. Synthesis and Characterization of $\pi$-Extended Triangulene. J. Am. Chem. Soc. 2019, 141 (27), 10621-10625. 
(27) Su, J.; Telychko, M.; Hu, P.; Macam, G.; Mutombo, P.; Zhang, H.; Bao, Y.; Cheng, F.; Huang, Z.-Q.; Qiu, Z.; Tan, S. J. R.; Lin, H.; Jelínek, P.; Chuang, F.-C.; Wu, J.; Lu, J. Atomically Precise Bottom-up Synthesis of $\pi$-Extended [5]Triangulene. $S c i . A d v .2019,5$ (7), eaav7717.

(28) Banhart, F.; Kotakoski, J.; Krasheninnikov, A. V. Structural Defects in Graphene. ACS Nano 2011, 5 (1), 26-41.

(29) Cretu, O.; Krasheninnikov, A. V.; Rodríguez-Manzo, J. A.; Sun, L.; Nieminen, R. M.; Banhart, F. Migration and Localization of Metal Atoms on Strained Graphene. Phys. Rev. Lett. 2010, 105 (19), 196102.

(30) Terrones, H.; Terrones, M.; Hernández, E.; Grobert, N.; Charlier, J.C.; Ajayan, P. M. New Metallic Allotropes of Planar and Tubular Carbon. Phys. Rev. Lett. 2000, 84 (8), 1716-1719.

(31) Lahiri, J.; Lin, Y.; Bozkurt, P.; Oleynik, I. I.; Batzill, M. An Extended Defect in Graphene as a Metallic Wire. Nat. Nanotechnol. 2010, 5 (5), 326-329.

(32) Yazyev, O. V.; Louie, S. G. Electronic Transport in Polycrystalline Graphene. Nat. Mater. 2010, 9 (10), 806-809.

(33) Ternes, M.; Heinrich, A. J.; Schneider, W.-D. Spectroscopic Manifestations of the Kondo Effect on Single Adatoms. J. Phys. Condens. Matter 2008, 21 (5), 053001

(34) Baumgarten, M.; Yüksel, T. Synthesis and Optical Properties of Novel Blue Fluorescent Conjugated Polymers. Phys. Chem. Chem. Phys. 1999, 1 (8), 1699-1706.

(35) Gross, L.; Mohn, F.; Moll, N.; Liljeroth, P.; Meyer, G. The Chemical Structure of a Molecule Resolved by Atomic Force Microscopy. Science 2009, 325 (5944), 1110-1114

(36) Kichin, G.; Weiss, C.; Wagner, C.; Tautz, F. S.; Temirov, R. Single Molecule and Single Atom Sensors for Atomic Resolution Imaging of Chemically Complex Surfaces. J. Am. Chem. Soc. 2011, 133 (42), $16847-$ 16851

(37) Pisani, L.; Chan, J. A.; Montanari, B.; Harrison, N. M. Electronic Structure and Magnetic Properties of Graphitic Ribbons. Phys. Rev. B 2007, 75 (6), 064418.

(38) Ovchinnikov, A. A. Multiplicity of the Ground State of Large Alternant Organic Molecules with Conjugated Bonds. Theor. Chim. Acta 1978, 47 (4), 297-304.

(39) Palma, C.-A.; Awasthi, M.; Hernandez, Y.; Feng, X.; Müllen, K.; Niehaus, T. A.; Barth, J. V. Sub-Nanometer Width Armchair Graphene Nanoribbon Energy Gap Atlas. J. Phys. Chem. Lett. 2015, 6 (16), 32283235 .

(40) Hirjibehedin, C. F.; Lutz, C. P.; Heinrich, A. J. Spin Coupling in Engineered Atomic Structures. Science 2006, 312 (5776), 1021-1024.

(41) Frota, H. O. Shape of the Kondo Resonance. Phys. Rev. B 1992, 45 (3), 1096-1099. 
Table of Contents artwork
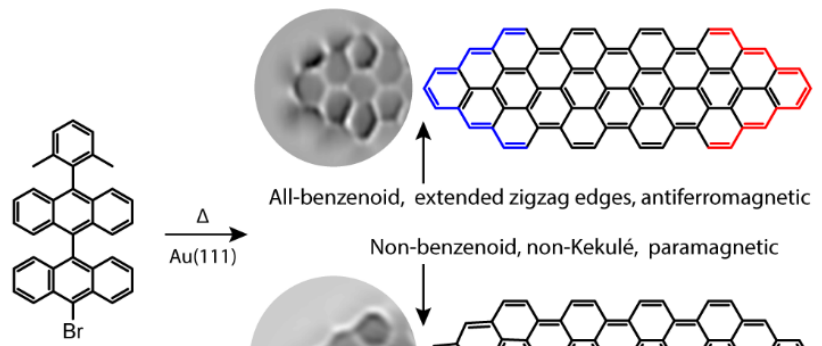

All-benzenoid, extended zigzag edges, antiferromagnetic

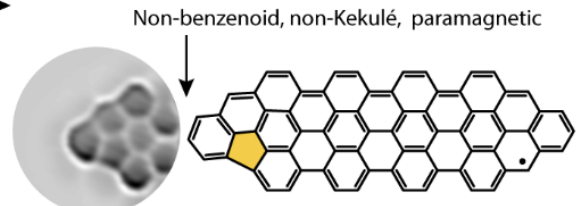

